\title{
Targeted treatment of idiopathic pulmonary fibrosis: one step at a time
}

\author{
Luca Richeldi ${ }^{1,2}$
}

Affiliations: ${ }^{1}$ Academic Unit of Clinical and Experimental Sciences, University of Southampton, Southampton, UK. ${ }^{2}$ NIHR Southampton Respiratory Biomedical Research Unit, University Hospital Southampton, Southampton, UK.

Correspondence: Luca Richeldi, National Institute for Health Research, Southampton Respiratory Biomedical Research Unit, Mailpoint 813, E Level, South Academic Block, University Hospital Southampton NHS Foundation Trust, Tremona Road, Southampton, S016 6YD, UK. E-mail: L.Richeldidsoton.ac.uk

-

@ERSpublications

Targeting CTGF in a selected group of IPF patients: a further step towards personalised treatments in IPF http://ow.ly/YMtEP

On October 21, 1999, physicians involved in the care of patients affected by idiopathic pulmonary fibrosis (IPF) read with enthusiastic surprise in the pages of the New England Journal of Medicine that this deadly disease could not only be stopped, but in fact be reverted by injecting interferon gamma-1b subcutaneously three times a week [1]. In an attempt to provide a mechanistic substrate to the impressive clinical results, this trial focused on two specific molecules, transforming growth factor- $\beta 1$ and connective tissue growth factor (CTGF), as the relevant therapeutic molecular targets, responsible for the functional improvement observed. In fact, the transcription levels of these two genes were significantly reduced in transbronchial biopsies obtained from some of the trial patients after 6 months of interferon gamma-1b treatment [1]. At the time of publication of this study, we didn't know (and we still do not know) if at least some of these 18 patients were representing a specific IPF subgroup (or endotype). As in the words of Niels Bohr, winner of the Nobel Prize in Physics in 1922, "prediction is very difficult, especially if it's about the future". Thus, we had to wait for two large placebo-controlled trials to be completed, involving several hundreds of patients being randomised [2,3], before knowing that the hopes for the possibility of reverting IPF with this drug had vanished; interferon gamma-1b is no longer considered an option for IPF patients. Nonetheless, the question of whether the original 1999 study identified a specific subgroup of "responders" still remains without a definitive answer.

Over recent years, thousands of IPF patients have been enrolled in different phases of clinical trials, and more and more the drugs tested, in particular in early-phase clinical trials, have been targeting specific molecular pathways [4-6]. Among these, CTGF might now be back on stage. In this issue of the European Respiratory Journal, RAGHU et al. [7] report the results of a prospective, open-label, phase 2 trial in which 89 IPF patients received, every 3 weeks for a total of 45 weeks, two different doses of FG-3019, an intravenous, fully humanised, monoclonal antibody, specifically designed to target CTGF. This trial follows the supportive results of pre-clinical studies in a mouse model of lung fibrosis, showing reversal of radiation-induced lung fibrosis after treatment with FG-3019, and the supportive findings of an open-label phase $1 \mathrm{~b}$ dose-escalation trial [8]. The aim of this further phase 2 open-label trial was ambitious: reverting (not stopping, not reducing) lung fibrosis in IPF, something similar to what we saw in the original small group of patients treated with interferon gamma-1b.

With the aim of identifying a group of patients more likely to respond to this approach, the study population was carefully selected; however, this did not result in an excessive screen failure rate, as in other recent trials [9]. Moreover, in contrast with most IPF trials completed so far, the radiological extent of disease on chest high-resolution computed tomography (HRCT) was arbitrarily pre-defined, with

Received: Feb 222016 | Accepted: Feb 222016

Conflict of interest: Disclosures can be found alongside the online version of this article at erj.ersjournals.com

Copyright @ERS 2016 
reticulation between $10 \%$ and $50 \%$ and honeycombing of $<25 \%$ of the whole lung. To be eligible for inclusion, patients had to show an objective progression of disease, either radiologically, functionally or symptomatically, in the period preceding the screening. Additionally, the extent of emphysema could not be greater than that of fibrosis on HRCT. These and other inclusion criteria, apparently restrictive, should be put into the context of the specific molecular mechanism of action of the drug under investigation and the attempt to target a population with a significant amount of potentially reversible fibrotic changes in the lung. It is therefore rational to think that patients without objective deterioration or with extensive irreversible destruction of the lung (i.e. honeycombing) will not benefit from treatment with an anti-CTGF drug. Interestingly, in the 1999 interferon gamma-1b study, patients with "end-stage" pulmonary fibrosis were excluded from the study [1].

The efficacy end-points of the study included computer-aided assessment of CT imaging. Although probably not yet ready for the prime time in clinical practice [10], this measure was carefully standardised in the trial, representing at this moment probably the best tool to quantify in a noninvasive way the amount of fibrosis in the lung. Although the HRCT protocol used in this trial, including quality controls and standardisation of reconstruction algorithms and of lung volumes, will probably not translate easily into clinical practice, it is still a significant step forward along the way to identifying reliable imaging tools to assess the efficacy of anti-fibrotic drugs.

As with any other trial, this study has limitations. The first and main limitation is the choice of design: a single-arm study was chosen, and not a more powerful, convincing, two-arm study including a placebo group. However, this insurmountable limitation is partly compensated by the precise identification of the study population, carefully selected to maximise the possibility of gaining the ambitious scope of fibrosis reversal. Secondly, the inclusion criteria were modified halfway through the completion of the study. Although a potential major flaw in any clinical trial, these changes seem to be justifiable and potentially very useful for the design of future trials. They were based on the observation that almost half of the patients with lower predicted forced vital capacity (FVC) prematurely discontinued from the study. These patients might have been too sick to contribute to a study requiring patients to travel often to clinical sites for infusions, blood drawings and imaging. Also, the criteria for disease progression were loosened based on the findings of the first cohort, in order to increase the signal in the trial. Is the study design potentially restricting the pool of patients eligible for treatment with FG-3019? Yes, although hopefully not in a restrictive, negative way, but with the aim of identifying a truly "responsive" population for the next phase of drug development. At the end of the process, ideally it will be possible to "give the right drug to the right patient at the right time", in line with the principles of precision medicine and with the expectations of IPF patients and their families [11].

This trial provided further evidence that FG-3019 has an acceptable safety profile, with most discontinuing patients having, not surprisingly, more severe disease. However, as Winston Churchill said once, "however beautiful the strategy, you should occasionally look at the results". Although efficacy should always be regarded as a secondary end-point in phase 2 trials, the results were supportive of FG-3019 being effective in IPF, at least in those selected for the trial and in particular in those with baseline FVC values $>55 \%$ predicted. One third of patients had either stable or improved extent of lung fibrosis, as assessed by HRCT. These 16 "responders" are, so far, among the few IPF patients demonstrating convincing evidence of fibrosis regression. Although overall most patients progressed in their disease, about one in five had an objective benefit from treatment. As in the two recent positive phase 3 trials leading to approval of new drugs [9, 12], none of the patient-related outcome measures showed any benefit, even in patients with an objective improvement in lung fibrosis. This is something that the sponsors and the investigators in charge of designing the next steps of FG-3019 development should keep in mind. The results of this study can be seen from two different angles: they might be considered a major advancement, if we consider the fact that so far no drug has been able to show consistent reversal of lung fibrosis in IPF; conversely, they can be seen as marginal, since only a minority of a highly selected patient population had a clear benefit. Of course, the lack of a placebo trial limits our ability to interpret the results, but nonetheless the presence of a subgroup of patients showing a reduction of the fibrotic changes in their lung is exciting.

The fact that, to the best of current knowledge, none of the two currently approved IPF treatments are targeting CTGF poses a promising basis for a future placebo-controlled trial combining FG-3019 with either pirfenidone and/or nintedanib [13]. However, an open question remains: which specific population of patients should be targeted to prove the efficacy of FG-3019 in the next clinical trial, a highly selected or a broader group? And which tools will be used to identify the right phenotype and the right endotype for the drug? These are crucial, challenging, exciting open questions, which will be relevant for any future trial in IPF. Answering them will require the coordinated, maximalised and global effort of the IPF community. 


\section{References}

1 Ziesche R, Hofbauer E, Wittmann $\mathrm{K}$, et al. A preliminary study of long-term treatment with interferon gamma- $1 \mathrm{~b}$ and low-dose prednisolone in patients with idiopathic pulmonary fibrosis. N Engl J Med 1999; 341: 1264-1269.

2 Raghu G, Brown KK, Bradford WZ, et al. A placebo-controlled trial of interferon gamma-1b in patients with idiopathic pulmonary fibrosis. N Engl J Med 2004; 350: 125-133.

3 King TE Jr, Albera C, Bradford WZ, et al. Effect of interferon gamma-1b on survival in patients with idiopathic pulmonary fibrosis (INSPIRE): a multicentre, randomised, placebo-controlled trial. Lancet 2009; 374: 222-228.

4 Richeldi L, Costabel U, Selman M, et al. Efficacy of a tyrosine kinase inhibitor in idiopathic pulmonary fibrosis. N Engl J Med 2011; 365: 1079-1087.

5 Raghu G, Martinez FJ, Brown KK, et al. CC-chemokine ligand 2 inhibition in idiopathic pulmonary fibrosis: a phase 2 trial of carlumab. Eur Respir J 2015; 46: 1740-1750.

6 Wilkes DS, Chew T, Flaherty KR, et al. Oral immunotherapy with type V collagen in idiopathic pulmonary fibrosis. Eur Respir J 2015; 45: 1393-1402.

7 Raghu G, Scholand MB, de Andrade J, et al. FG-3019 anti-connective tissue growth factor monoclonal antibody: results of an open-label clinical trial in idiopathic pulmonary fibrosis. Eur Respir J 2016; 47: 1481-1491.

8 Lipson KE, Wong C, Teng Y, et al. CTGF is a central mediator of tissue remodeling and fibrosis and its inhibition can reverse the process of fibrosis. Fibrogenesis Tissue Repair 2012; 5: Suppl. 1, S24.

9 King TE Jr, Bradford WZ, Castro-Bernardini S, et al. A phase 3 trial of pirfenidone in patients with idiopathic pulmonary fibrosis. N Engl J Med 2014; 370: 2083-2092.

10 Hansell DM, Goldin JG, King TE Jr, et al. CT staging and monitoring of fibrotic interstitial lung diseases in clinical practice and treatment trials: a position paper from the Fleischner Society. Lancet Respir Med 2015; 3: 483-496.

11 Bonella F, Wijsenbeek M, Molina-Molina M, et al. European IPF Patient Charter: unmet needs and a call to action for healthcare policymakers. Eur Respir J 2016; 47: 597-606.

12 Richeldi L, du Bois RM, Raghu G, et al. Efficacy and safety of nintedanib in idiopathic pulmonary fibrosis. $N$ Engl J Med 2014; 370: 2071-2082.

13 Wells A. Combination therapy in idiopathic pulmonary fibrosis: the way ahead will be hard. Eur Respir J 2015; 45: $1208-1210$. 